\title{
Spontaneous splenic rupture secondary to lymphoma: Two case reports
}

\author{
Semih Gülle $₫$, Harun Akar® \\ Department of Internal Medicine, University of Health Sciences, Izmir Tepecik Training and Research Hospital, Izmir, Turkey
}

\begin{abstract}
Spontaneous splenic rupture (SSR) is a dramatic abdominal emergency that requires immediate diagnosis and prompt surgical intervention to ensure survival. The most common causes of SSR are infections, hematologic malignancies, and cystic lesions of the spleen. Although the spleen is often involved in hematological malignancies, splenic rupture is a rare condition. We present two cases of non-traumatic splenic rupture secondary to B-cell lymphoma infiltration. Emergency splenectomy is the basis for treatment of splenic rupture.

Keywords: Lymphoma, splenectomy, splenic rupture.
\end{abstract}

Spontaneous splenic rupture (SSR) is associated with high morbidity and mortality rates in non-traumatic patients, rarely resulting in atypical clinical features and delayed onset. The complaints of the patients range among a broad spectrum and acute abdomen is rarely encountered. Appropriate imaging methods have an important place in the differential diagnosis of these cases.

Spontaneous splenic rupture rarely requires histologic evaluation for diagnosis, and usually occurs in a diseased spleen, therefore it is often called "pathological spontaneous rupture", while such cases are called "true spontaneous rupture. ${ }^{[1]}$

Diseases that commonly cause SSR are often hemato-oncological diseases. In addition, various clinical manifestations such as infectious diseases (infectious mononucleosis and malaria), congenital splenic lesions, acute and chronic pancreatitis, hamartoma, hemangioma, cyst, autoimmune diseases, hemolytic anemia, pregnancy, amyloidosis, and portal hypertension may also cause SSR. ${ }^{[2]}$

The prevalence of SSR due to hematological diseases has been reported as $1 \%$ in the literature. Primer splenic lymphoma is the first order of hematological causes of spontaneous splenic rupture. In this article, we aim to present two patients who experienced spontaneous splenic rupture due to primary splenic lymphoma following various clinical manifestations.

\section{CASE REPORT}

Case 1- A 77-year-old woman was admitted to our clinic with complaints of increased consciousness and abdominal pain in the last 24 hours after the last six months of weight gain, swelling in her stomach and increased complaints of loss of appetite. She did not mention any complaints related to defecation or urinary tract changes. She did not describe any recent trauma or fall. She was diagnosed with hypertension

Received: January 25, 2020 Accepted: February 26, 2020 Published online: August 25, 2020

Correspondence: Semih Gülle, MD. Sağlık Bilimleri Üniversitesi, İzmir Tepecik SUAM, İç Hastalıkları Kliniği, 35180 Yenişehir, Konak, İzmir, Türkiye. Tel: +90 541 - 6442466 e-mail: semih.gulle@hotmail.com 
and used amlodipine (10 mg) and nebivolol (5 mg) once a day. On physical examination, the patient was pale, alert, and tachypneic. Her blood pressure was $85 / 45 \mathrm{mmHg}$ and heart rate $>140 \mathrm{bpm}$. The patient had deep palpation pain in the left lower quadrant of the abdomen spreading to the left shoulder. Electrocardiogram and chest X-ray were normal. Biochemical test results were normal except for lactate dehydrogenase (LDH) $(1,520 \mathrm{U} / \mathrm{L}$; normal range: $95-195 \mathrm{U} / \mathrm{L})$. In the complete blood count, white blood cell (WBC): 14.2 (4.0-10.0 $\times$ $\left.10^{3} / \mathrm{mm}\right) \mathrm{Hgb}: 11 \mathrm{gr} / \mathrm{dL}(12.3-15.7 \mathrm{~g} / \mathrm{dL})$ and platelet (PLT): $110,000\left(130-400 \times 10^{3} / \mathrm{mm}^{3}\right)$ were detected. Peripheral blood smear was performed for the bicytopenia, no schistoside or atypical cells. Coombs tests were all negative.

Increased spleen volume, heterogeneous appearance, and free fluid in the abdomen were observed in the ultrasonography (USG) examination due to the patient's abdominal pain and pain in the upper left side. Contrast-enhanced computed tomography (CT) was taken to clarify the patient's diagnosis and showed a solid mass appearance in the spleen and concurrent rupture (Figure 1 and Figure 2).

Considering the SSR in the patient, emergency operation was performed after obtaining consent. During the operation, a mass of $6 \times 8 \mathrm{~cm}$ and a laceration of $4 \mathrm{~cm}$ were observed in the posterolateral part of the spleen. Splenectomy was applied to the patient who had $1,500 \mathrm{~mL}$ of aspirated blood content from the abdomen.
Appropriate antimicrobial prophylactic vaccines (depot penicillin, acti-Hib ${ }^{\circledR}$, Pneumo $23^{\circledR}$ ) for prophylactic treatment were administered as a single dose 24 hours postoperatively. Severe sepsis and acute respiratory distress syndrome (ARDS) developed in the postoperative period in our intensive care unit.

The patient died on the seventh day after the operation. Pathologic evaluation reported that the primary spleen lymphoma was diffuse large B-cell lymphoma, occupying the entire spleen. Immunohistochemistry revealed that CD20, CD79a, MUM1, BCL6, FOXP1, and p53 (partially) were positive, while $\mathrm{CD} 3, \mathrm{CD} 10$, and BCL2 were negative.

Case 2- A 66-year-old male patient was admitted to our outpatient clinic with fatigue, fever, and nausea for the past one month. During the last 15 days, he had fever and nausea with chills at intervals, and decreased appetite. It was learned that the patient had a left knee prosthesis related to an accident 10 years ago and that he was using $1,000 \mathrm{mg}$ metformin twice a day for four years.

The physical examination of the patient showed exhaustion and pale appearance. Blood pressure was $125 / 70 \mathrm{mmHg}$, pulse $100 \mathrm{bpm}$ and fever $38.6^{\circ} \mathrm{C}$. There was pain with deep palpation in the upper left abdomen. Results of biochemical tests revealed LDH 450 U/L (normal range: 95-195 U/L), aspartate transaminase (AST) (155 IU/L; normal range: 0-35 U/L) and alanine aminotransferase (ALT) (144 IU/L;
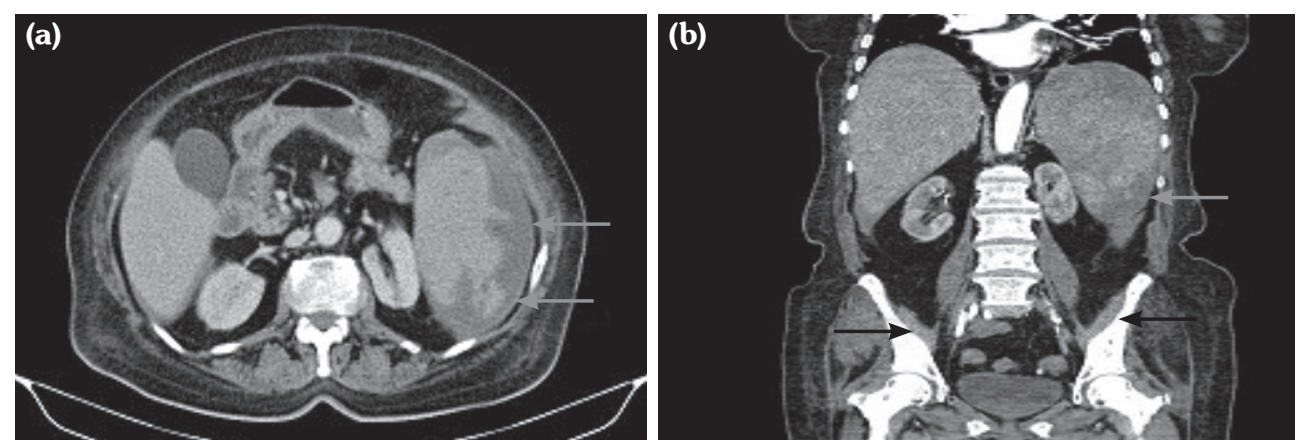

Figure 1. (a) The spleen is abnormally large and heterogeneity in the parenchyma is evident. Subcapsular field is compatible with hematoma and solid mass (gray arrows). (b) It is noted that the subcapsular hematoma in the vicinity of the paracolic gutter extends (gray arrow) and pelvic free fluid (black arrows). 

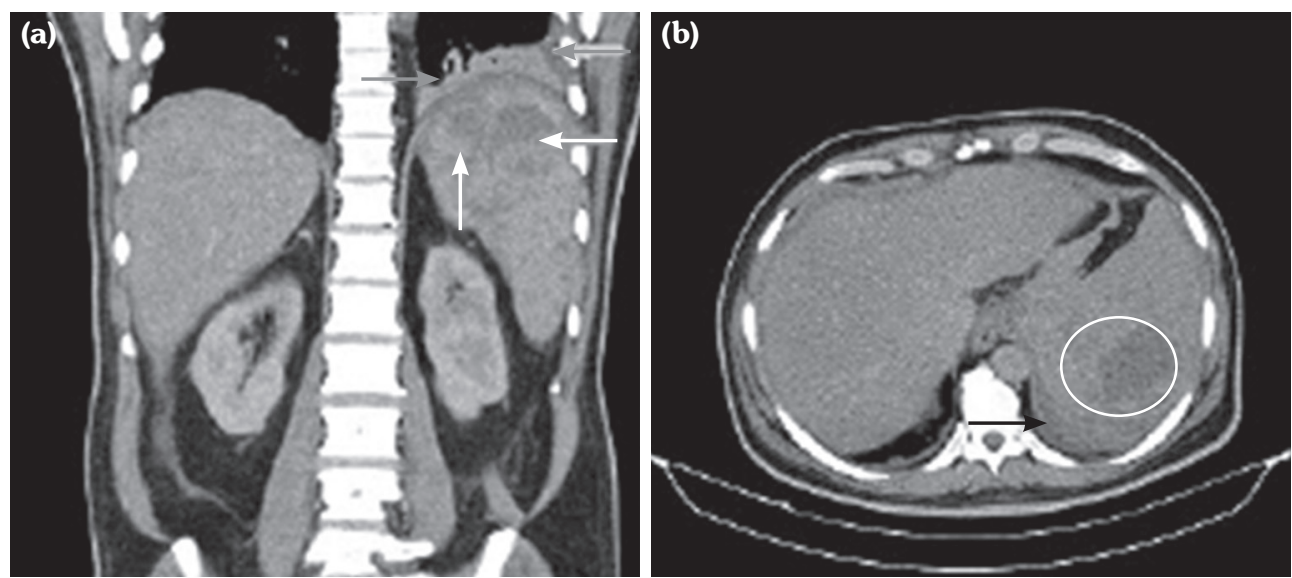

Figure 2. (a) The spleen is normally seen as large and heterogeneous (white arrow) and pleural cannulae reaching $1.5 \mathrm{~cm}$ in the left hemithorax and in the vicinity of the atelectasis in the vicinity (gray arrows). (b) Splenic vein is present in the tortuous as far as possible and there is hypodense area near the upper left pole in the parenchyma (white ring). Subcapsular hematoma is remarkable (black arrow).

normal range: 3-36 $\mathrm{U} / \mathrm{L})$ were found to be increased by four times.

Viral hepatitis markers were negative. His complete blood count revealed WBC: 10,300; PLT: 195,000; and hemoglobin (Hgb) $11.2 \mathrm{gr} / \mathrm{dL}$. There was no evidence of active infection in the physical examination, laboratory, or imaging studies of the patient who was having recurrent fevers after admission. Patient peripheral blood cultures were obtained. None of the cultures were positive. Abdomen ultrasound was performed revealing spleen dimensions of $19 \times 10 \mathrm{~cm}$ and heterogeneous appearance of the spleen.

The cause of the heterogeneous appearance of the spleen with recurrent fevers and cardiac involvement with primary diagnosis of septic embolism of the spleen. The patient underwent transthoracic echocardiography (TTE) and transesophageal echocardiography (TEE). There was no vegetation finding compatible with endocarditis.

Contrast-enhanced abdominal CT was performed to exclude a possible infarction, abscess, or bleeding in the spleen after a 24-hour Hgb decline of $2 \mathrm{gr} / \mathrm{dL}$. Abdominal CT revealed that the patient's spleen capsule could not be clearly distinguished. The appearance that could be compatible with hemorrhage was widespread (Figure 3 and Figure 4) SSR was considered together with these findings in a patient who had no history of trauma to the abdominal region.

Emergency splenectomy was performed with the consent of the patient. During the operation, ruptured spleen and widespread bleeding were observed in the abdomen. It was stated that no pathology was observed in other abdominal organs.

The vital signs of the patient improved after the operation and there was no deterioration of blood values throughout follow-up. A single dose of appropriate antimicrobial prophylactic vaccines (depot penicillin, acti-Hib ${ }^{\circledR}$, Pneumo $23^{\circledR}$ ) was administered to the patient at 12 hours. The patient was healed and discharged on postoperative day six.

The spleen material of the patient was evaluated as diffuse large B-cell lymphoma. The patient was followed up by the hematology department. The patient is currently progressionfree and undergoing follow-up in the fourth cycle of rituximab plus cyclophosphamide, doxorubicin, vincristine, and prednisone (R-CHOP) chemotherapy protocol.

\section{DISCUSSION}

Spontaneous spleen rupture is a very rare cause of acute abdomen that leads to serious clinical consequences. ${ }^{[3,4]}$ It often occurs as a 
congestion that develops after the infective and hematological infiltration of the spleen. It is rarely encountered in patients undergoing anticoagulant therapy or who have bleeding disorders. ${ }^{[5]}$ The frequency of spontaneous splenic rupture is not clearly known. There are few case reports in the literature. The vast majority of these patients have benign and infectious disorders. Other causes include hematologic malignancies and other rare diseases. ${ }^{[6,7]}$

Spontaneous splenic rupture is a life-threatening complication that requires early diagnosis and treatment. The incidence is not precisely known because it is a rare clinical condition. The spleen is one of the first organs to be affected in hematologic malignancies, because it is a vital organ of the immune system. ${ }^{[6]}$

Elderly age, male sex, and splenomegaly are the most common causes of spleen rupture. Splenomegaly is especially attributed as the main factor causing rupture. It is noteworthy that our patients have rupture without severe splenomegaly. Coagulation disturbances due to lymphoblastic infiltration, spleen infarction, coagulation disorders causing intrasplenic and/or subcapsular hemorrhage, and thrombocytopenia are the most frequently suspected factors in the etiopathogenesis of hematologic causes of SSR. ${ }^{[8]}$

Increased rate of development of splenomegaly also increases the risk of rupture due to sudden increase in capsule distention. Early diagnosis of pathological spleen rupture is a significant factor in determining the patient's prognosis. Based on physical examination, the history is structured to be recognized by imaging methods such as USG and CT, after an initial suspicion of this diagnosis, helps to reach early diagnosis. ${ }^{[9]}$ Sudden onset of stomach, left upper quadrant and left shoulder pain (Kehr sign) without trauma are the most common clinical complaints.

The fact that one of our patients had complaints of fever and fatigue for the last one month is noteworthy, as SSR may present with particularly different clinical entities. Due to high mortality rates, proper treatment should be planned as soon as spleen rupture is detected.

Although rare cases of patients treated with conservative treatment according to clinical status have been reported, early consideration of surgical intervention should be considered as a rational approach considering the benefit/loss ratio. ${ }^{[10]}$ In addition, the most important point that should not be overlooked in the postoperative period is providing appropriate prophylaxis against infections that may develop after splenectomy. ${ }^{[11]}$ It is recommended that the prophylactic agents (depot penicillin, encapsulated bacterial vaccines) be administered within 72 hours after surgery.

Spontaneous splenic rupture is the first symptom of a hematologic malignancy and can be confronted as a life-threatening complication. When SSR is considered a possible diagnosis in an appropriate clinical setting, early diagnosis and emergency splenectomy, as well as prompt pathological evaluation are strongly recommended.

\section{Declaration of conflicting interests}

The authors declared no conflicts of interest with respect to the authorship and/or publication of this article.

\section{Funding}

The authors received no financial support for the research and/or authorship of this article.

\section{REFERENCES}

1. Orloff MJ, Peskin GW. Spontaneous rupture of the normal spleen; a surgical enigma. Int Abstr Surg 1958;106:1-11.

2. Biswas S, Keddington J, McClanathan J. Large B-cell lymphoma presenting as acute abdominal pain and spontaneous splenic rupture; a case report and review of relevant literature. World J Emerg Surg 2006;1:35.

3. Adachi K, Arima D, Hosaka A, Kiriu T, Sakashita $\mathrm{K}$, Kojima A, et al. A non-traumatic splenic rupture leads to diagnosis of underlying abnormality. Lancet 2014;384:1820.

4. Elvy A, Harbach L, Bhangu A. Atraumatic splenic rupture: a 6-year case series. Eur J Emerg Med 2011;18:124-6.

5. Ingle $\mathrm{SB}$, Ingle $\mathrm{CR}$. Splenic lymphoma with massive splenomegaly: Case report with review of literature. World J Clin Cases 2014;2:478-81.

6. Szotkowski T, Szotkowska R, Pikalova Z, Tichy T, Flodr P, Tichy M, et al. Spontaneous splenic rupture in two patients with hematologic malignancy. Leuk Res 2007;31:S100.

7. Mason KD, Juneja SK. Spontaneous rupture of the spleen as the presenting feature of the blastoid variant of mantle cell lymphoma. Clin Lab Haematol 2003;25:263-5.

8. Sozutek A, Kupeli AH, Saban B, Topuz S, Ozdemir $\mathrm{M}$, Citil R. Pathologic splenic rupture related to 
non-hodgkin lymphoma. J Acad Emerg Med Case Reports 2014;5:193-6.

9. Unal E, Onur MR, Akpinar E, Ahmadov J, Karcaaltincaba M, Ozmen MN, et al. Imaging findings of splenic emergencies: a pictorial review. Insights Imaging 2016;7:215-22.

10. Bosch N, Renteria AS, Quillen K, Brauneis D,
Santilli J, Kim D, et al. Nonoperative Management of Spontaneous Splenic Rupture in a Patient with LightChain Amyloidosis: A Case Report. J Vasc Interv Radiol 2015;26:1578-80.

11. Dahyot-Fizelier C, Debaene B, Mimoz O. Management of infection risk in asplenic patients. Ann Fr Anesth Reanim 2013;32:251-6. 